\title{
Concurrence Entre Banques Commerciales Et Opérateurs De Téléphonie Mobile En Afrique Subsaharienne
}

\author{
Florent Jean Désiré Kabikissa, \\ Université Marien Ngouabi \\ Faculté des Sciences Économiques, République du Congo
}

Doi:10.19044/esj.2019.v15n34p235 URL:http://dx.doi.org/10.19044/esj.2019.v15n34p235

\section{Résumé}

L'objet de cet article est d'étudier, sous forme d'un jeu, la concurrence entre une banque et un opérateur de téléphonie mobile sur un marché où les services sont différenciés horizontalement. L'hypothèse retenue dans cette étude est que les firmes se font une concurrence en localisation géographique, et le choix de localisation sera interprété comme un choix dans un espace de produits. Pour ce faire, le modèle de Hotelling a été mobilisé pour appréhender d'une part, l'impact de la concurrence sur l'industrie bancaire en Afrique, et d'autre part, les avantages stratégiques dont bénéficient les deux acteurs. Il ressort de cette étude qu'il n'existe pas d'équilibre de Nash en stratégie pure en jeu simultané, mais qu'une structure de «leader-follower» conduit à l'équilibre et permet aux firmes de réaliser des profits positifs. Banques et opérateurs de téléphonie mobile ont donc un avantage mutuel à coopérer, sous des formes diverses qui restent à définir, plutôt qu'à se faire concurrence.

Mots-clés: Concurrence, Banques Commerciales, Opérateurs De Téléphonie Mobile, Afrique Sub-Saharienne 


\title{
Competition Between Commercial Banks and Mobile Telephone Operators in Subsaharan Africa
}

\author{
Florent Jean Desire Kabikissa, \\ Université Marien Ngouabi \\ Faculté des Sciences Économiques, République du Congo
}

\begin{abstract}
This paper focuses on studying, in the form of a game, the competition between a bank and a mobile operator in a market where services are differentiated horizontally. The hypothesis used in this study shows that firms compete with one another within a geographic location. The choice of location will be interpreted using a product space. To achieve this, the Hotelling model was mobilized to understand, on the one hand, the impact of competition on the banking industry in Africa, and on the other hand, the strategic advantages enjoyed by both actors. This study shows that there is no Nash equilibrium in pure strategy in regard to simultaneous play, but that a leader-follower structure leads to equilibrium and allows firms to make positive profits. Banks and mobile phone operators therefore have a mutual advantage to cooperate, in various forms that are yet to be defined, rather than competing with each other.
\end{abstract}

Keywords: Competition, Commercial Banks, Mobile Operators, Sub-Saharan Africa

\section{Introduction}

Dans la plupart des Pays Africains, on observe une inadéquation des systèmes financiers face aux exigences du développement (Hugon, 1990). Parmi les facteurs considérés comme principales causes des mauvaises performances des économies africaines, on note généralement la difficulté d'accès aux services bancaires et financiers pour une majorité de la population (Kiendrebeogo \& Minea, 2013). Celle-ci est exclue de compte bancaire, de moyens de paiements ou du crédit de première nécessité, parce qu'elle n'est pas en mesure de fournir des garanties matérielles suffisantes ${ }^{21}$. Mais aussi, parce que les coûts de sélection, de surveillance et d'incitation sont très importants pour les banques (Fall, 2011). Selon la Banque Africaine de

21 En Afrique Sub-Saharienne, seuls 12\% d'adultes sont bancarisés (Banque Africaine de Développement, 2013, p.1). 
Développement (2013), seuls $12 \%$ de la population adulte africaine détiendraient un compte bancaire. Pourquoi une telle situation ? La banque africaine est-elle adaptée à son environnement social, culturel tout autant qu'aux besoins du développement ? (Le Noir, 1997). Autant de questions que se sont posé d'autres économistes, mais qui ne feront pas l'objet de cette étude.

Pour Hugon (1991), le système financier africain s'est développé culturellement, économiquement et socialement en rupture avec la société civile, dont les agents sont exclus de ce système. Ainsi, faute de moyens (économiques, financiers et technologiques) pour développer leurs économies et combattre la pauvreté, les Pays Africains se sont vu imposer par la Banque Mondiale et le Fonds monétaire international à la fin des années 1980, une série de réformes, parmi lesquelles, la libéralisation et la privatisation de leurs systèmes financiers, l'amélioration du cadre réglementaire et institutionnel, dans le but de les rendre plus efficaces, et d'attirer les investissements étrangers. Pour les experts des institutions de Bretton Woods, ces réformes sont censées instaurer, selon Stiglitz (2002), un climat qui attire les investissements étrangers, qui favorise la croissance et un large accès aux services financiers (dépôts et prêts) dans l'espoir de réduire substantiellement la pauvreté.

Le paysage bancaire africain a été profondément renouvelé depuis la grave crise de solvabilité et de liquidité de la fin des années 1980-début des années 1990. De nouveaux acteurs ont supplanté les banques historiques telles que la Banque Internationale pour l'Afrique de l'Ouest (BIAO), la Banque Nationale de Paris (BNP), le Crédit Lyonnais, la Société Générale, la Citibank et la Belgolaise etc. Les banques étatiques ou de développement ont disparu, des normes réglementaires plus contraignantes ont été imposées par les autorités de tutelle. Il en est donc ressorti un renforcement des dispositifs de contrôle des banques et, plus généralement, une prise de conscience davantage marquée vis-à-vis du risque (Diop, 2015).

Depuis l'an 2000, les nouvelles banques panafricaines qui se sont développées en Afrique Sub-Saharienne, ainsi que les banques locales évoluent dans un environnement très concurrentiel. À côté des banques occidentales apparaissent de nouveaux acteurs tels que les groupes bancaires marocains et les opérateurs de téléphonie mobile.

Malgré les réformes de leurs systèmes financiers, les «banques commerciales africaines », ne se localisent que dans des zones urbaines (quartiers d'affaires ou riches) et industrielles (minières ou agricoles) où il existe un certain pouvoir d'achat. Les zones rurales et pauvres du souscontinent, où le besoin social des services financiers est important, sont exclues de la base de clientèle des principales institutions financières. Cela s'explique selon Eber (2000), par le fait que les agences localisées dans les quartiers difficiles et pauvres sont peu rentables en raison des faibles revenus 
d'une clientèle potentiellement plus risquée, et l'exclusion géographique résulterait donc d'une volonté des banques de réduire leur risque, leurs coûts et d'améliorer leur rentabilité.

En réponse à cette exclusion, les opérateurs de la téléphonie mobile soucieux de s'adapter aux contextes locaux, proposent depuis la fin des années 2000 , le service « mobile-money ${ }^{22}$ » dont les premières expériences africaines ont vu le jour en 2007, au Kenya. Ces opérateurs se comportent en « banques sans agences » et apparaissent moins comme de nouveaux entrants sur le secteur bancaire que comme un modèle de diversification des banques généralistes (Marceau, 2011). Ils proposent un service mobile de paiement, d'achat de crédits téléphoniques, de paiement dans les commerces, de règlement de factures, de collecte de l'épargne et de transfert d'argent.

L'industrie bancaire africaine connaît depuis plusieurs années de profondes transformations, la déréglementation, les turbulences accrues, l'apparition de nouvelles formes de concurrence, l'adoption des nouvelles technologies etc. (Hamel \& Prahalad, 1994). Ces forces sont à l'origine des restructurations sans précédent qui affectent aussi bien la structure industrielle que l'organisation des firmes à l'image de rapprochements entre banques sous forme de fusion-acquisition ou contrats. Des acteurs auparavant étrangers aux métiers de la banque, en quête de diversifications, notamment les opérateurs des télécommunications, s'immiscent dans l'industrie bancaire en s'appuyant sur leurs compétences propres dont les banques africaines ne disposent pas et seraient dans l'incapacité de reproduire dans l'immédiat (Lehmann-Ortega \& Roy, 2009). Ils apportent sur ce marché, des innovations frugales, en adoptant les technologies des télécommunications (Short Message System [SMS]), créées dans les économies les plus avancées pour les adapter à des nouveaux usages et dans un contexte local (Chaix \& Torre, 2015). Ils s'appuient aussi sur la logique du « low-cost», en créant des nouveaux marchés, non compétitifs et indépendants du marché existant, dont les principaux clients sont des individus qui, jadis n'avaient ni les moyens financiers, ni les moyens intellectuels pour utiliser les services bancaires et financiers (Kim \& Mauborgne, 1997). Ces opérateurs mobiles jouent un rôle déterminant dans la collecte de l'épargne et le financement de populations pauvres au point de devenir une nouvelle forme d'intermédiation financière.

Pourtant, la théorie économique justifie l'existence des banques commerciales dans la sphère économique par la présence des asymétries d'information et les coûts de transaction prohibitifs liés à l'échange direct sur le marché (Eber, 2000; Fall, 2011). En Afrique, la présence des opérateurs mobiles dans l'industrie bancaire serait alors, une des conséquences de

\footnotetext{
22 «Le mobile-money correspond à de la monnaie électronique émise par un système privatif, que possède le consommateur » (Leboucher, 2016).
} 
l'inefficacité des banques quant à l'évaluation et la réduction des coûts afférents à certains types de projets. Depuis quelques années, l'Afrique est devenue le théâtre d'une concurrence acharnée entre les banques et les institutions non financières, notamment, les opérateurs de la téléphonie mobile. Cette concurrence concerne davantage la collecte de l'épargne que l'offre de crédits et se justifie selon Chaix \& Torre (2015) d'une part, par une forte adhésion de la population africaine à la téléphonie mobile : son taux de pénétration est passé de $22,9 \%$ à $89,4 \%$ entre 2005 et 2013. D'autre part, par des faibles coûts liés aux opérations effectuées auprès des opérateurs mobiles. La technologie peut entraîner une réduction des coûts et des risques liés aux transactions, permettant ainsi d'effectuer de petites transactions et de rendre davantage de ménages et d'entreprises économiquement rentables (Beck \& Cull, 2014). Par exemple, les coûts de transaction d'un transfert d'argent à partir d'un téléphone mobile seraient de l'ordre de 0,08 dollars US, tandis qu'ils avoisineraient les 4 dollars pour la même opération dans des succursales bancaires (Grimes, 2010). Mais aussi, parce que les techniques de mobilemoney ont des coûts d'adaptation négligeables et sont faciles à appréhender et à appliquer, même dans un contexte d'analphabétisme. Cette simplicité les rend plus attractives, et s'oppose aux procédures lourdes et contraignantes associées à l'ouverture d'un compte bancaire.

Le présent article s'intéresse aux effets de la concurrence entre les banques commerciales et les opérateurs mobiles sur le marché bancaire africain. L'arrivée des opérateurs mobiles favorise-t-elle la concurrence, donc la baisse des prix et un large accès aux services financiers ? Ou au contraire renforce-t-elle le pouvoir des firmes ? Pour ce faire, cet article est structuré comme suit : la première partie présente le modèle théorique et les différentes étapes de la concurrence, tandis que la deuxième partie présente les principaux résultats de l'étude ainsi que leur interprétation. Une conclusion termine l'article.

\section{Modèle Et Présentation Des Caractéristiques}

Partout en Afrique, les opérateurs mobiles redéfinissent les règles du jeu au sein de l'industrie bancaire, ils imposent aux banques traditionnelles un nouveau type de concurrence, en captant une partie de leur clientèle potentielle, grâce à la maîtrise de leurs infrastructures. Cette concurrence comporte un aspect inédit par rapport à l'analyse classique de la concurrence dans l'industrie bancaire, dans la mesure où les contraintes auxquelles font face les deux agents, sont relativement différentes (Dumans, 2003). Les acteurs en concurrence sont de types différents, et leurs différences portent à la fois, sur leur logique d'intermédiation et sur leurs contraintes géographiques. Pour un banquier, la proximité de la clientèle est essentielle pour déterminer sa zone de chalandise tandis qu'un opérateur mobile a juste 
besoin d'un téléphone, qu'il utilise comme vecteur de flux financiers pour pallier le manque de canaux formels de transaction bancaire (Bounie et al., 2013). La banque doit supporter des coûts fixes engendrés par la construction d'agences (appelé ici coût logistique) pour desservir ses clients. En revanche, l'opérateur mobile n'a pas besoin d'agences pour servir ses clients et ne supporte pas ce type des coûts. Il crée simplement des points « d'entrée et de sortie » gérés par des agents indépendants, mais certifiés, c'est-à-dire, les endroits où les agents certifiés permettent aux clients de services mobiles de retrouver des liquidités quand ils le souhaitent, en convertissant, soit le code en espèces, soit des espèces en code lorsque ces derniers veulent constituer une épargne ${ }^{23}$.

Par ailleurs, la banque propose une tarification composée d'une part du prix du service acheté par le client et d'autre part, d'un prix du transport qui est variable, car il dépend de la distance parcourue par le client jusqu'à l'agence qui est à la charge du client. Par contre l'opérateur mobile propose une tarification qui ne comporte que le prix du service et ce prix est identique quel que soit l'éloignement géographique du client, et ne dépend que du montant de la transaction. Le présent modèle s'intéresse au comportement concurrentiel des firmes à politiques de prix donnés. Le modèle le plus proche du notre est celui de Dumans (2003), qui étudie la concurrence entre le commerce électronique et le commerce traditionnel dans lequel, les agents ne sont pas tous soumis aux mêmes contraintes.

Dans ce papier il est fait l'hypothèse que les firmes se font réellement une concurrence en localisation géographique et le choix de localisation sera interprété comme un choix dans un espace de produits. Pour décrire le processus concurrentiel entre les banques et les opérateurs mobiles, le modèle de Hotelling (1929) sera extrapolé à l'industrie bancaire, en raison du type de concurrence étudié. La structure oligopolistique de l'industrie bancaire africaine est plus marquée par une concurrence par les prix que par les quantités et se caractérise par une différentiation horizontale des produits, liée notamment à des différences de type entre les firmes (Weill, 1998). Le modèle de Hotelling permet en effet de mieux prendre en compte les deux aspects de la concurrence, en termes des prix et de différentiation des produits.

\section{1. Le Modèle}

Le modèle analyse la concurrence entre deux firmes, la banque (A) et un opérateur mobile (B) qui offrent un bien homogène sur le marché et se font concurrence en produits et en localisations. Il se présente sous forme d'un jeu à deux étapes dans lequel les firmes choisissent leurs localisations dans un

\footnotetext{
${ }^{23}$ Les transferts se font par le biais d'un code, lorsque le bénéficiaire d'un code veut transformer la monnaie électronique qu'il a reçue, il transfère son code à un intermédiaire certifié qui le transforme en monnaie liquide.
} 
premier temps, avant de choisir dans un deuxième temps leurs prix respectifs en se livrant une concurrence à la Bertrand. La résolution du modèle s'effectue en «backward induction ». Les consommateurs sur le marché considéré sont distribués uniformément sur le segment [0,1], qui représente l'espace des caractéristiques du produit, dans lequel les firmes sont localisées à l'intérieur du segment. Un consommateur situé à l'extrême du segment achète le produit qui lui permet de minimiser sa désutilité, mesurée par le coût psychique perçu à choisir un produit différent de son produit idéal plus le prix d'achat. La fonction de coût est supposée être de la forme $t$. $(\check{x})$ où $\tilde{x}$ et $t(t \geq 0)$ représentent respectivement le consommateur marginal (indifférent) et le degré d'exigence des consommateurs concernant la caractéristique du produit. L'introduction des caractéristiques signifie que les produits ne sont pas parfaitement substituables et les firmes peuvent être en situation de monopole sur leur marché local. En effet, si $t$ est nul, cela implique que la caractéristique du produit n'a aucune influence et la concurrence est parfaite. Au contraire, plus $t$ est grand, plus le coût psychique de la désutilité liée à la consommation d'un produit non idéal est important. Le coût marginal de production est supposé être nul sans perte de généralité dans la classe des fonctions de coût linéaires et, le marché est couvert, c'est-à-dire que les prix sont suffisamment faibles pour que tous les consommateurs achètent chacun une unité du bien à la firme qui lui propose le prix le plus bas, dont ils tirent tous la même utilité. Lorsque les deux firmes ont la même localisation, qu'elles sont toutes en activité, il peut être admis qu'une fraction quelconque non nulle des consommateurs choisit la firme A, et que les autres choisissent la firme B. S'il n'achète pas sa caractéristique idéale, le consommateur supporte une désutilité égale à $t$. $\tilde{x}, \mathrm{~s}^{\prime}$ il achète à la firme $\mathrm{A}$, alors que celle-ci est égale à $t .(1-\tilde{x}) \mathrm{s}$ 'il s'adresse à la firme $\mathrm{B}$. Cette désutilité est interprétée comme étant le coût de la distance, qui sépare une spécification «idéale » d'un produit à la spécification réelle relative à la variété disponible la plus proche.

Dans ce modèle, tout consommateur est supposé définir une variété « idéale », mais comme les goûts sont diversifiés et le nombre de variété infini, les firmes en concurrence ne peuvent satisfaire toutes les demandes. Faute de pouvoir trouver leur variété idéale, les consommateurs choisissent alors la variété qui se rapproche le plus de leur spécification idéale (Siroën, 1987). Cette distance psychique entre la variété disponible et la variété idéale est donc nulle pour le consommateur qui, par chance, bénéficie de la variété idéale mais elle est maximale pour le consommateur indifférent à deux variétés également distantes de son idéal. Il est ainsi défini par :

$$
p_{A}+t \cdot \tilde{x}=p_{B}+t \cdot(1-\check{x})
$$


L'équation (1) signifie que le consommateur indifférent est situé entre les deux firmes et elle peut être réécrite de la façon suivante $: \frac{1}{2}+\frac{p_{B}-p_{A}}{2 t}=\tilde{x}$

Dans ce modèle, il est donc question d'étudier à court terme, les effets d'une variation de variétés des biens sur l'intensité de la concurrence, les prix, les profits des firmes etc.

\section{2. Résultats Et Stratégies Des Firmes}

Si l'une des firmes ne s'écarte pas trop du prix généralisé de sa concurrente $\left(p_{A}<p_{B}-\mathrm{t}\right)$ ou $\left(p_{B}<p_{A}-t\right)$, les fonctions de demande sont données par les expressions suivantes :

$$
\begin{aligned}
& D_{A}\left(p_{A}, p_{B}\right)=\left\{\begin{array}{c}
1 \text { si } p_{A}<p_{B}-t \\
\tilde{x}=\frac{1}{2}+\frac{p_{B}-p_{A}}{2 t} \text { si } p_{B}-t \leq p_{A} \leq p_{B}+t \\
0 \text { si } p_{A}>p_{B}+t
\end{array}\right. \\
& D_{B}\left(p_{A}, p_{B}\right)=\left\{\begin{array}{c}
1 \text { si } p_{B}<p_{A}-t \\
1-\tilde{x}=\frac{1}{2}-\frac{p_{B}-p_{A}}{2 t} \text { si } p_{A}-t \leq p_{B} \leq p_{A}+t \\
0 \text { si } p_{A}-t<p_{B}
\end{array}\right.
\end{aligned}
$$

$D_{A}\left(p_{A}, p_{B}\right)$ et $D_{B}\left(p_{A}, p_{B}\right)$ sont les demandes adressées respectivement à la firme $\mathrm{A}$ et à la firme $\mathrm{B}$. Une fois que les firmes ont choisi leurs localisations, la seule variable stratégique dont elles disposent à court terme, c'est le prix. Selon les hypothèses retenues dans cette étude, il sera analysé dans un premier temps un jeu en séquentiel (la banque est considérée comme un leader établi sur le marché $)^{24}$ pour toute distribution possible des consommateurs sur l'espace des caractéristiques. Deux cas de figure vont être distingués, selon que les firmes choisissent la même localisation (c'est-à-dire elles sont très proches) ou selon qu'elles s'éloignent l'une de l'autre.

(i) : lorsque les deux firmes choisissent la même localisation :

$\mathrm{Ce}$ cas traduit une situation de duopole et les firmes se font concurrence en prix. Elles choisissent leurs prix de manière à maximiser leur profit à prix de l'autre donné. La zone de duopole n'est définie que si $\mathrm{D}_{A}\left(\mathrm{p}_{A}, \mathrm{p}_{B}\right) \geq$ $0, \forall \mathrm{A}, \mathrm{B}$, tel que $\mathrm{A} \neq \mathrm{B}$. À partir des (3) et (4), on déduit les expressions des profits :

$$
\begin{aligned}
& \Pi^{A}\left(p_{A}, p_{B}\right)=p_{A} . D_{A}\left(p_{A}, p_{B}\right)=\left(p_{A}\right)\left(\frac{p_{B}-p_{A}+t}{2 t}\right) \\
& \Pi^{B}\left(p_{A}, p_{B}\right)=p_{B} . D_{B}\left(p_{A}, p_{B}\right)=\left(p_{B}\right)\left(\frac{p_{A}-p_{B}+t}{2 t}\right)
\end{aligned}
$$

Les conditions de premier ordre d'optimisation permettent d'obtenir :

$$
\begin{aligned}
& p_{A}^{*}\left(p_{B}\right)=\frac{p_{B}+t}{2} \\
& p^{*}{ }_{B}\left(p_{A}\right)=\frac{p_{A}+t}{2}
\end{aligned}
$$

\footnotetext{
${ }^{24}$ Dans un jeu séquentiel, le leader joue toujours en premier.
} 
La meilleure réponse de chacune des firmes à la stratégie de l'autre est définie par :

$$
p^{*}=p^{*}=t
$$

À partir de (3) et (4), les demandes adressées aux deux firmes peuvent être exprimées comme suit : $D^{*}{ }_{A}=D_{B}^{*}=\frac{1}{2}$

Les deux firmes se partagent donc le marché à parts égales et réalisent des profits positifs : $\Pi_{A}^{*}=\Pi_{B}^{*}=p_{A}{ }^{*} D_{A}{ }^{*}=p_{B} D^{*}{ }_{B}=\frac{t}{2}$

Proposition 1 : si les firmes se localisent à proximité du centre, il n'existe pas d'équilibre de Nash en stratégies pures du sous-jeu en prix, car les prix d'équilibre, s'ils existent doivent satisfaire les conditions du premier ordre d'optimisation.

La preuve: si $t=0$, l'équilibre du sous-jeu obtenu n'est pas un équilibre de Nash, est un équilibre de Bertrand, puisque les profits des deux firmes sont nuls. Or si les firmes s'éloignaient l'une de l'autre, leurs profits augmentent avec $t\left(\frac{\partial \Pi}{\partial t}>0\right)$.

Ce qui montre que l'équilibre obtenu est un équilibre local, or tout équilibre de Nash est global. Pour que l'équilibre $\left(p^{*}{ }_{A}, p^{*}{ }_{B}\right)$ soit un équilibre de Nash, il faut que les deux agents économiques choisissent une stratégie de manière que $\left(p^{*}{ }_{A}, p^{*}{ }_{B}\right)$ soit la meilleure réponse quelle que soit la stratégie utilisée par l'un des concurrents. Ce qui implique :

$\Pi_{A}^{*}\left(p^{*}{ }_{A}, p^{*}{ }_{B}\right) \geq \Pi_{A}^{*}\left(p_{A}, p^{*}{ }_{B}\right), \forall p_{A} \neq p_{A}^{*}$ dans l'ensemble des stratégies $P$ $\left(P\right.$ étant l'ensemble des prix) et $\Pi_{B}^{*}\left(p^{*}{ }_{A}, p^{*}{ }_{B}\right) \geq \Pi_{B}^{*}\left(p^{*}{ }_{A}, p_{B}\right), \forall p_{B} \neq p_{B}^{*}$ dans $P$.

Ainsi, si les firmes se rapprochent et se mettent l'une à côté de l'autre, elles cessent de se différencier et vendent des produits homogènes, qui sont des substituts parfaits. Ce qui implique une concurrence parfaite entre elles. $\mathrm{Ce}$ comportement est favorable à l'inclusion financière, puisque la concurrence en prix va favoriser la baisse des prix et un meilleur accès aux services financiers. Mais, la dynamique de la concurrence en prix les poussera à fixer les prix au niveau du coût marginal, ce qui exclut toute possibilité d'un profit positif $^{25}$. Un tel comportement ne correspond pas à un équilibre de Nash, parce que les deux firmes ont la possibilité en adoptant une stratégie différente, d'améliorer chacun son profit. Ce qui revient à jouer sa stratégie dominante si elle en a une. La stratégie dominante dans ce jeu, est celle où les deux firmes maximisent simultanément leurs profits, en fixant respectivement des prix qui satisfont les conditions du premier ordre d'optimisation.

(ii) Lorsque les deux firmes ne choisissent pas la même localisation : ${ }^{25}$ La firme qui supporte des coûts de production très élevés par rapport à l'autre risque de se voir
évincer du marché. 
Cette situation caractérise un duopole avec produits différenciés (ce qui implique que $\mathrm{D}_{\mathrm{A}}\left(\mathrm{p}_{\mathrm{A}}, \mathrm{p}_{\mathrm{B}}\right) \neq \mathrm{D}_{\mathrm{B}}\left(\mathrm{p}_{\mathrm{A}}, \mathrm{p}_{\mathrm{B}}\right) \geq 0$ et $\mathrm{p}_{\mathrm{A}}^{*}>0$, $\left.\mathrm{p}^{*}{ }_{\mathrm{B}}>0\right)$, les deux firmes en décidant de se localiser sur des endroits différents évitent une concurrence en prix et tiennent captifs leurs clients. Ce comportement stratégique pourrait être néfaste à la dynamique d'inclusion financière. Il peut empêcher que certains clients du système mobile basculent vers le système bancaire, limitant ainsi l'accès aux banques qu'à une population plus aisée. Il est clair que $\Pi^{*}{ }_{A}$ et $\Pi^{*}{ }_{B}$ augmentent avec $t$. En se différentiant, les firmes améliorent leurs profits, en réalisant des profits positifs. Cette observation invalide le principe de différentiation minimale de Hotelling, qui stipule que les firmes seraient désireuses de se rapprocher l'une de l'autre pour s'établir au centre du marché.

En se différentiant, chacune des firmes maximise son profit et cette stratégie est une stratégie dominante dans le sens de Nash. Ce comportement est dû au fait que lorsqu'un bien n'est différencié que par sa seule localisation, il donne naissance à deux biens qui sont des compléments stratégiques (Combes et al., 2006).

Proposition 2 : dans un jeu séquentiel, à stratégie donnée de l'autre joueur, il est toujours possible d'obtenir un profit positif en choisissant un produit très différent et en fixant un prix strictement positif suffisamment faible.

Preuve : lorsque les deux firmes sont localisées aux deux extrémités du segment, pour qu'elles soient toutes les deux actives, il faut que $\left[p_{B}-\right.$ $\left.p_{A}\right]<t$, sinon l'une des concurrentes servirait la totalité du marché. Comme l'opérateur mobile possède un hinterland constitué de la population pauvre et démunie, la banque peut fixer son prix de sorte que : $p_{A}<p_{B}-t$, elle augmenterait ainsi sa demande des services financiers et mettra l'opérateur mobile hors du marché, car son prix sera plus faible que celui pratiqué par son concurrent et tous les consommateurs appartenant à l'hinterland de l'opérateur mobile choisiront de s'approvisionner auprès de la banque. Mais, une telle stratégie n'est ni réalisable ni profitable si: $\Pi_{A}^{*}\left(p^{*}{ }_{A}, p_{B}^{*}\right) \geq \Pi_{A}^{*}\left(p_{A}, p_{B}^{*}\right)$.

Comme les deux firmes sont très éloignées l'une de l'autre, la baisse des prix que doit supporter la banque pour approvisionner tout le marché est considérable et s'avère non profitable. Toutefois, $\left(p_{A}, p_{B}^{*}\right)$ n'est pas un équilibre de Nash puisque le profit réalisé par l'opérateur mobile est nul, ce qui implique que $p^{*}{ }_{B}$ n'est pas la meilleure réponse de l'opérateur mobile à $p_{A}$. En effet, lorsque les coûts psychiques de la désutilité sont élevés, la dimension géographique domine les autres, l'éloignement protège les firmes et infléchit la concurrence. Chacune approvisionne son seul marché local, ce qui contredit le principe de différenciation minimale de Hotelling. 


\section{Interprétation Des Résultats Et Discussion \\ II.1. Une Concurrence Spatialisée}

Hotelling a conçu en 1929, un modèle de concurrence en prix et en localisations que nous pouvons interpréter comme décrivant le processus concurrentiel entre deux firmes, l'une offrant un produit standardisé et l'autre un produit spécifique conformément à l'analyse développée dans les sections précédentes. D'Aspremont et al. (1979) ont montré qu'il n'existe pas d'équilibre quand les firmes sont localisées l'une à côté de l'autre. Cependant, lorsque le jeu se déroule par étapes, si l'une des firmes s'engage avant l'autre dans le choix de sa localisation, l'existence d'équilibre peut être restaurée. En effet, si la banque évite de se localiser au centre comme dans le modèle de base de concurrence horizontale, la raison en est que l'opérateur mobile sera à son tour incité de se positionner aussi au centre, acceptant ainsi une concurrence frontale. En effet, lorsqu'ils choisissent exactement le même produit, les deux firmes chercheront à se différencier pour éviter une concurrence en prix, qui peut s'avérer dévastatrice pour elles. C'est ce qui est généralement appelé en économie, le principe de différenciation de Hotelling, que Combes et al. (2006) expliquent par la conjonction des deux effets : l'effet aire du marché et l'effet de concurrence en prix. Le premier qui, à prix donné, incite chaque firme à se rapprocher de son concurrent afin d'augmenter ses parts de marché, tandis que le second pousse les firmes à se séparer pour restaurer leurs marges. Le second effet domine toujours le premier. Cela pousse alors les deux firmes à se différencier.

Par ailleurs, comme le choix de localisation pour la banque est plus rigide que celui de prix, il s'avère que dans l'industrie bancaire en Afrique, les banques et les opérateurs mobiles ne sont pas soumis aux mêmes contraintes. Ces derniers créent de nouveaux marchés non compétitifs en s'appuyant sur leurs propres ressources dont les banques ne disposent pas dans l'immédiat. Les contraintes géographiques font que le choix du produit et du prix soit plus flexible pour les opérateurs mobiles que pour les banques. Par ailleurs, les économies d'échelle à réaliser font que les banques revoient rarement leurs prix face à leurs concurrents locaux puisqu'elles n'ont pas la totale maîtrise de leurs prix, qui sont en partie influencés par le niveau du taux directeur des Banques Centrales.

Le processus concurrentiel sur le marché bancaire africain se déroule comme si les banques jouaient en premier, en choisissant un produit standard alors que l'opérateur mobile choisit ensuite, un produit plus spécifique dans la première étape. Dans la deuxième étape, l'opérateur mobile, bien qu'il soit un follower, joue en premier en choisissant son prix et la banque joue en second et choisit un prix plus élevé. En effet, la concurrence spatialisée entre banques et opérateurs mobiles peut s'avérer néfaste du point de vue du bien-être collectif, elle pousse les firmes en concurrence à adopter des comportements 
abusifs, en fixant des prix plus élevés que leurs coûts de production, confisquant ainsi tout surplus aux consommateurs pauvres des services financiers. À terme, cela risque de limiter l'accès aux services financiers qu'à une frange de la population ayant un pouvoir d'achat. Ainsi, comme le notent Guellec \& Ralle (2003), en Afrique Sub-Saharienne où les institutions ne sont pas soutenues, lorsque le marché est livré à lui-même, il semble dans l'incapacité de conduire spontanément au bien-être collectif ; d'où la nécessité d'un organe de coordination pour le réglementer afin d'éviter tout dérapage.

\section{II.2. Les Limites De L'inclusion Financière}

Si la concurrence entre les banques et les opérateurs mobiles a permis d'élargir la surface d'intermédiation financière et d'augmenter l'inclusion financière en Afrique Sub-Saharienne, force est de reconnaître que cette concurrence reste très spatialisée. Les zones rurales, les plus peuplées et les plus pauvres du sous-continent, où le besoin social des services financiers est le plus important, sont dépourvues des infrastructures financières de base. Les services financiers bon marché comme les moyens de paiement ou l'épargne sont quasi-inexistants. Dans ces zones, selon la Banque Africaine de Développement (2013), les banques éprouveraient du mal à desservir les populations pauvres non bancarisées en raison essentiellement des coûts élevés d'établissement d'un réseau d'agences et de l'étroitesse des marges associées aux opérations des banques en faveur des pauvres. En se localisant sur leurs marchés traditionnels, les banques et les opérateurs mobiles tiennent captifs leurs clients respectifs.

Grâce aux paiements mobiles, les opérateurs mobiles peuvent davantage contribuer à la promotion du développement économique en facilitant les opérations de marché. La plateforme mobile apporte aux zones géographiquement reculées, des solutions novatrices pratiques sous forme de services bancaires au-delà des agences. Ces services sont aujourd'hui offerts aux populations des couches socioéconomiques les plus basses. L'accès massif de la population subsaharienne à la téléphonie mobile permettra d'accélérer le développement de ces nouveaux services.

\section{Conclusion}

Cette étude a analysé les conséquences de la concurrence entre les banques traditionnelles et les opérateurs mobiles à travers le modèle de Hotelling. Le constat est que ce type de concurrence ne pose aucun problème de stabilité ou d'équilibre si le processus concurrentiel se déroule sous forme d'un jeu séquentiel avec un leader établi. Lorsque les deux firmes choisissent la même localisation, c'est-à-dire lorsqu'elles sont géographiquement très proches, elles se font concurrence en prix et choisissent leurs prix de manière à maximiser leur profit. Quoiqu'un tel comportement ne corresponde pas à un 
équilibre de Nash, la stratégie dominante dans ce cas est celle où les deux firmes maximisent simultanément leurs profits, en fixant respectivement des prix qui satisfont les conditions du premier ordre d'optimisation. La concurrence en prix va favoriser la baisse des prix et un meilleur accès aux services financiers ; ce qui est favorable à l'inclusion financière. En revanche, lorsque les deux firmes ne choisissent pas la même localisation et s'éloignent l'une de l'autre, elles évitent de se livrer une concurrence en prix et tiennent captifs leurs clients. Ce résultat qui est une stratégie dominante dans le sens de Nash, invalide le principe de différentiation minimale de Hotelling, et révèle que chacune des firmes maximise son profit en choisissant un produit très différent et en fixant un prix strictement positif suffisamment faible. Mais, ce comportement stratégique peut être néfaste à la dynamique d'inclusion financière en empêchant certains clients du système mobile de basculer vers le système bancaire formel.

La concurrence entre banques et opérateurs de téléphonie mobile en Afrique Sub-Saharienne est sensible à la localisation, et reste très spatialisée. Les banques africaines s'engagent plus fortement sur leurs marchés traditionnels et refusent de se mettre au milieu du marché, en se rapprochant de leurs concurrents et imposant aux opérateurs mobiles une concurrence sous forme d'un jeu dans lequel il y a lutte pour le second coup afin que l'équilibre du marché soit tel que le leader, c'est-à-dire les banques, choisissent un produit plus générique à un prix plus élevé alors que les opérateurs mobiles choisissent un produit plutôt spécialisé à un prix faible.

Pourtant, des initiatives de coopération peuvent être envisagées par la banque qui a intérêt non seulement à exploiter la base installée de l'opérateur pour bénéficier des innovations et des compétences de son partenaire, mais aussi pour bénéficier du levier procuré par l'offre simplifiée de l'opérateur mobile. Les banques africaines gagneraient donc à proposer aux opérateurs mobiles les conditions d'un partenariat qui les incitent tous à coopérer.

Ce travail théorique ne reste toutefois qu'une étape. Il pourrait être prolongé dans deux directions: par une analyse en termes de bien-être, consistant à comparer le surplus net obtenu dans une situation de coopération entre les banques et les opérateurs mobiles d'une part, et à celui d'une situation concurrentielle d'autre part.

\section{References:}

1. Banque Africaine de Développement (2013). «Inclusion et intégration financière à travers les paiements et transferts mobiles » in Actes de l'atelier sur «Le renforcement de l'intégration financière par la réglementation judicieuse des paiements mobiles transfrontaliers: opportunités et défis », 29-30 mars 2012, Mumbai, Inde, 66 p. 
2. Beck T., Cull R. (2014). « Les systèmes bancaires en Afrique subsaharienne : un état des lieux », Revue d'Économie Financière, Vol. 4, $\mathrm{n}^{\circ} 116$, pp. 43-56.

3. Bounie D., Dimitrescu D. \& François A. (2013). «On the Effect of Mobile Phone on Migrant Remittances: A Closer Look at International Transfers », Electronic Commerce Research \& Applications, Vol. 12, $\mathrm{n}^{\circ}$ 3, pp. 280-288.

4. Chaix L., Torre D. (2015). « Le double rôle du paiement mobile dans les pays en développement », Revue Économique, Vol. 66, n 4, pp. 703-727.

5. Combes P., Mayer T. \& Thisse J.F. (2006). «Économie géographique : L'intégration des régions et des nations », Economica, Paris, $350 \mathrm{p}$.

6. D’Aspremont C., Gabszewics J. \& Thisse J.F. (1979). « On hotelling's "stability in competition", Econometrica, Vol. 47, pp. 1045-1050.

7. Diop S. (2015). «L'évolution du système bancaire en zone franc », Techniques Financières et Développement, vol. 121(4), pp. 59-69.

8. Dumans M.E. (2003). «Concurrence entre commerce électronique et commerce traditionnel », Revue Économique, Vol. 54, n 3, pp. 687696.

9. Eber N. (2000). « Sélection de clientèle et exclusion bancaire », Revue d'Économie Financière, $n^{\circ} 58$, pp. 79-96, Paris.

10. Fall F.S. (2011). «La complémentarité Banques/Microfinance dans les économies en développement : une perspective théorique », Revue d'Économie Industrielle, Vol. 133, n², pp. 31-56, Paris.

11. Grimes C. (2010). "Beyond "Check-the box" mobile banking and payment », Illinois Banker, pp. 13-22.

12. Guellec D., Ralle P. (2003). «Les nouvelles théories de la croissance », Paris, La Découverte, collection « Repères », 128 p.

13. Hamel G., Prahalad C.K. (1994). « Strategy as a Field of Study : Why Search for a New Paradigm ? », Strategic Management Journal, vol. $15, \mathrm{n}^{\circ} 5$, pp. 5-16.

14. Hotelling H. (1929). « Stability in Competition », Economic Journal, 39, pp. 41-57.

15. Hugon P. (1991). « Les politiques d'ajustement et le dualisme financier africain », Épargne Sans Frontière, TFD, n 23 , pp. 22-30.

16. Hugon P. (1990). «L'impact des politiques d'ajustement sur les circuits financiers informels africains » Tiers-Monde, Vol. 31, $\mathrm{n}^{\circ} 122$, pp. 325-349.

17. Kiendrebeogo Y., Minea A. (2013). « Accès aux services financiers et réduction de la pauvreté dans les PED », Revue Économique, Vol. 64, $\mathrm{n}^{\circ} 3$, pp. 483-493. 
18. Kim C., Mauborgne R. (1997). «Value Innovation : The Strategic Logic of High Growth », Harvard Business Review, Vol. 75, n 1, pp. 102-112.

19. Le Noir A. (1997). «La situation et les perspectives d'évolution des systèmes financiers d'Afrique noire francophone », Revue d'Économie Financière, $\mathrm{n}^{\circ}$ 41, pp. 169-177.

20. Lehmann-Ortega L., Roy P. (2009). «États des lieux : Les stratégies de rupture », Revue Française de Gestion, Vol. 35, n 197, pp. 113126.

21. Marceau G. (2011). «Banques en ligne : du mimétisme au «ebuisness model » bancaire », Gestion 2000, Vol. 28, n 4, pp. 49-65, Paris.

22. Siroën J.M. (1987). «La discrimination des prix dans un cadre de concurrence spatiale », Revue d'Économie Industrielle, Vol. 42, $n^{\circ} 1$, pp. 1-15.

23. Stiglitz J.E. (2002). « La grande désillusion», Fayard, Paris, 324 p.

24. Weill L. (1998). « Concurrence et efficience dans la banque ». Revue Française d'Économie, Vol. 13, n ${ }^{\circ}$, pp. 101-127. 